\title{
Prevalence of Soil-Transmitted Helminths and Molecular Clarification of Hookworm Species in Ethnic Ede Primary Schoolchildren in Dak Lak Province, Southern Vietnam
}

\author{
Bui Khac Hung', Nguyen Van De ${ }^{2, *}$, Le Van Duyet ${ }^{3}$, Jong-Yil Chai ${ }^{4,5}$ \\ ${ }^{1}$ Krong Pac Hospital, Dak Lak Province, Vietnam, ${ }^{2}$ Hanoi Medical University, Hanoi, Vietnam, ${ }^{3}$ National Hospital of Tropical Diseases (NHTD), Hanoi, \\ Vietnam; ${ }^{4}$ Department of Parasitology and Tropical Medicine, Seoul National University College of Medicine, Seoul 03080, Korea; ${ }^{5}$ Korea Association \\ of Health Promotion, Seoul 03722, Korea
}

\begin{abstract}
To know the infection status of helminths in primary schoolchildren of southern parts of Vietnam, we performed an epidemiological study in Krong Pac district, Dak Lak Province, Vietnam. A total of 1,206 stool specimens were collected from ethnic Ede schoolchildren in 4 primary schools in 2015 and examined by the Kato-Katz technique. In addition, stool cultures were done by the Harada-Mori method to obtain hookworm larvae and then to clarify the species of hookworms infected. The results showed that the helminth infection rate was $25.0 \%$, including $2.0 \%$ Ascaris lumbricoides, $0.33 \%$ Trichuris trichiura, and $22.8 \%$ hookworm infections. The average intensity of infection was 102.0 eggs per gram of feces (EPG) for Ascaris, 36.0 EPG for Trichuris, and 218.0 EPG for hookworms. ITS1 gene sequences of the hookworm larvae were identical with those of Necator americanus (100\% homology) reported in GenBank. It has been confirmed in this study that the hookworm, $N$. americanus, is a dominant helminth species infected in primary schoolchildren of a southern part of Vietnam. Public health attention is needed for control of hookworm infections among schoolchildren in surveyed areas of Vietnam.
\end{abstract}

Key words: Ascaris lumbricoides, Trichuris trichiura, hookworm, soil-transmitted helminth, prevalence, intensity, schoolchildren, Vietnam

\section{INTRODUCTION}

It is estimated that $576-740$ million individuals are infected with hookworms worldwide [1,2]. Of the infected individuals, about 80 million are severely affected [2]. The major etiology of hookworm infections is Necator americanus which is found in the Americas, sub-Saharan Africa, and Asia [3]. Ancylostoma duodenale is found in more scattered focal environments, namely Europe and the Mediterranean [3]. Most infected individuals are concentrated in sub-Saharan Africa, East Asia, and the Pacific Islands with each region having estimates of 198 million and 149 million infected individuals, respectively. Other affected regions include South Asia (59 million), Latin America and the Carribeans (50 million), and Middle East/ North Africa (10 million) [1]. In the United States, 95\% of hu-

\footnotetext{
- Received 7 May 2016, revised 7 July 2016, accepted 17 July 2016.

*Corresponding author (ngvdeyhn@gmail.com)

(C) 2016, Korean Society for Parasitology and Tropical Medicine

This is an Open Access article distributed under the terms of the Creative Commons Attribution Non-Commercial License (http://creativecommons.org/licenses/by-nc/4.0) which permits unrestricted non-commercial use, distribution, and reproduction in any medium, provided the original work is properly cited.
}

man hookworm cases are caused by N. americanus, primarily in young schoolchildren in economically deprived rural areas. Juvenile hookworms cannot survive freezing temperatures, so the highest prevalence occurs in areas with warmer temperatures and greater rainfall $[4,5]$. The greatest incidence of infections occurs in Asia and sub-Saharan Africa, especially in poverty-stricken areas with poor sanitation [3]. A. duodenale infections occur at a lesser rate and are seen primarily in Europe and the Mediterranean.

In Vietnam, several studies have been reported regarding the prevalence of hookworm infections [6-10]. In 2006, 65 million individuals were reported to be infected with helminths, including Ascaris lumbricoides, Trichuris trichiura, and hookworms. Hookworms are distributed in the whole country, and the prevalence was $68 \%$ in the south and $85 \%$ in the north [6]. In the northern part, 95-98\% of human hookworm cases were caused by N. americanus [7], whereas in the south there were no reports on this information. In Nghe An Province (in the north), the helminthic infection was $98 \%$, which included Ascaris infection $83.6 \%$, Trichuris infection $85.1 \%$, and hookworm infection $30.3 \%$ [8]. Identification of species for A. duo- 
denale or N. americanus by morphology of adult worms is easy, but using eggs is difficult; however, one can use the third-stage filariform larvae for identification $[4,5]$.

The present study was undertaken to determine the prevalence of soil-transmitted helminths, including Ascaris, Trichuris, and hookworms, among schoolchildren in 4 primary schools in an ethnic community Ede in Krong Pac district, Dak Lak Province, South Vietnam, using the Kato-Katz technique and stool cultures by the Harada-Mori method. In addition, the species of hookworms prevalent in this area was determined using a molecular analysis of the filariform larvae.

\section{MATERIALS AND METHODS}

\section{Stool examination and stool culture}

Stool examinations were performed on 1,206 schoolchildren using the Kato-Katz technique. In this technique, the smears were examined in a systematic manner, and the eggs of each species were reported to give the number of the eggs per gram of feces (EPG). Stool cultures were performed by the Harada-Mori method for 100 positive samples with hookworms, with the highest infection (incubating fecal material on a filter paper strip in a test tube containing water for the purpose of culturing and recovering hookworm larvae) for identification of the hookworm species (after 10 days from culture).

\section{Morphological identification of hookworm larvae}

Identification of hookworm species was done by the morphology of filariform larvae according to the key of Sasa et al. [11] reported in 1958 (Table 1).

\section{Identification of hookworm larvae by molecular method}

Species identification of hookworm larvae was also done by PCR using the gene ITS1 (internal transcribed spacer 1) of rDNA and comparison with these genes reported in GenBank. For DNA isolation, Qiagen kits (DNeasy Blood \& Tissue Kit, Qiagen Sciences, Germantown, Maryland, USA) were used for extraction of the total DNA from the hookworm N. americanus

Table 1. Identification key ${ }^{a}$ of hookworm species by the morphology of filariform larvae

\begin{tabular}{lll}
\hline Organ & Ancylostoma duodenale & Necator americanus \\
\hline Mouth & Dim and thin & Dark and exclamation mark \\
Tail & Straight tail & Bent tail \\
\hline
\end{tabular}

aSasa et al. [11]. in accordance with the manufacturer's protocol. In brief, worm homogenates were resuspended in $100 \mu \mathrm{l}$ of manufacturer's lysis buffer containing $>8 \mathrm{mM}$ EDTA, $>0.5 \%$ SDS, and $20 \mu \mathrm{l}$ proteinase $\mathrm{K}$, which was then incubated at $56^{\circ} \mathrm{C}$ for 30 min. Thereafter, $4 \mu \mathrm{l}$ RNase and $200 \mu \mathrm{l}$ lysis buffer were added and treated in accordance with the manufacturer's protocol (for a microfuge scale preparation).

\section{PCR amplification of ITS1}

PCR for amplification of the $250 \mathrm{bp}$ fragment of the ITS1 of Necator was performed in a $50 \mu \mathrm{l}$ volume. PCR reactions were performed in $10 \mathrm{mM}$ Tris-HCl (pH 8.4), $50 \mathrm{mM} \mathrm{KCl}, 3.0 \mathrm{mM}$ $\mathrm{MgCl}_{2}, 250 \mu \mathrm{M}$ each of dATP, dCTP, dGTP, and dTTP, and 50 pmol of each primer with $1 \mathrm{U}$ Taq polymerase (Promega, Madison, Wisconsin, USA). The following primers were used in separate reaction mixes: NC1 (5'-ACGTCTGGTTCAGGGTTGTT-3') and NC2 (5'-TTAGTTTCTTTTCCTCCGCT-3'); OB (5'-TATATTGCAACAGGTATTTTGGTAC- $3^{\prime}$ ) and NC2; NA (5'-ATGTGCACGTTATTCACT-3') and NC2 [13]. Amplification was first conducted for 25 cycles using the primer set NC1-NC2. Then, $2 \mu \mathrm{l}$ of each NC1-NC2 amplicon was transferred to a fresh tube containing the same PCR reaction buffer with the primer set OB-NC2 and another with the primer set NA-NC2, and amplified for another 35 cycles. Cycling was performed in a Genius Thermal Cycler (Techne, Cambridge, UK) using the following parameters: initial denaturation at $94^{\circ} \mathrm{C}$ for $5 \mathrm{~min}$, followed by 25 cycles ( 35 cycles in the second PCR) of $94^{\circ} \mathrm{C}$ for $30 \mathrm{sec}$ (denaturation), $55^{\circ} \mathrm{C}$ for $30 \mathrm{sec}$ (annealing), and $72^{\circ} \mathrm{C}$ for $30 \mathrm{sec}$ (extension), followed by a final extension at $72^{\circ} \mathrm{C}$ for $5 \mathrm{~min}$.

\section{Sanger sequencing}

Dideoxy sequencing was performed using BigDye ${ }^{\mathrm{TM}}$ Terminator Chemistry v. 3.1 (Applied Biosystems, Foster City, California, USA) according to the manufacturer's instructions, as described previously. Forward and reverse primers for N. americanus were used as sequencing primers (section 2.3) using the ABI 3130 Bioanalyzer (Applied Biosystems).

\section{Phylogenetic analysis}

Multiple sequence alignments were performed by using the ATGC software version 7.0.2 and the Clustal W program to determine the nucleotide and amino acid sequence similarities. Phylogenetic trees were constructed in MEGA 6.06 using the neighbor-joining (NJ) cluster algorithm with evolutionary distances estimated using the Kimura 2-parameter model; boot- 
Table 2. Helminthic infections in schoolchildren of 4 primary schools

\begin{tabular}{|c|c|c|c|c|c|c|c|c|c|}
\hline \multirow{2}{*}{ School code } & \multirow{2}{*}{ No. exam. } & \multicolumn{2}{|c|}{ Helminthic infection } & \multicolumn{2}{|c|}{ Ascaris } & \multicolumn{2}{|c|}{ Trichuris } & \multicolumn{2}{|c|}{ Hookworms } \\
\hline & & $(+)$ & $\%$ & $(+)$ & $\%$ & $(+)$ & $\%$ & $(+)$ & $\%$ \\
\hline 1 & 316 & 113 & 35.8 & 10 & 3.2 & 1 & 0.32 & 103 & 32.6 \\
\hline 2 & 298 & 97 & 32.6 & 7 & 2.4 & 1 & 0.34 & 89 & 29.9 \\
\hline 3 & 301 & 54 & 17.9 & 2 & 0.66 & 1 & 0.33 & 52 & 17.3 \\
\hline 4 & 291 & 37 & 12.7 & 5 & 1.7 & 1 & 0.34 & 31 & 10.7 \\
\hline Total & 1,206 & 301 & 25.0 & 24 & 2.0 & 4 & 0.33 & 275 & 22.8 \\
\hline
\end{tabular}
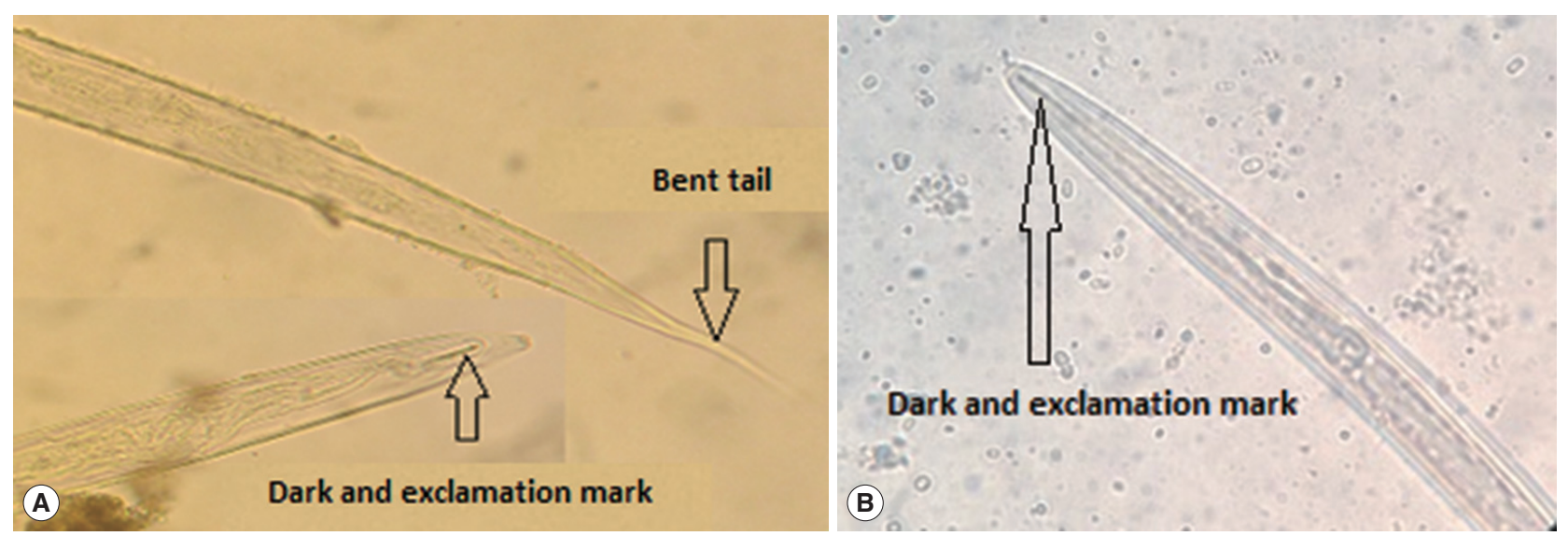

Fig. 1. Eggs (A: before culture; B: egg after hatching) and larvae of Necator americanus.

strapping was performed using 1,000 pseudo-replicates.

\section{RESULTS}

\section{Prevalence of helminths}

Out of 1,206 stool samples collected from schoolchildren and examined by the Kato-Kaz technique, the overall prevalence of helminth eggs was $25.0 \%$ (12.7-35.8\% by school), including 22.8\% hookworm infections (10.7-32.6\%), 2.0\% Ascaris infections (0.66-3.2\%), and $0.33 \%$ Trichuris infections (0.32-0.34\%) (Table 1). By gender, the prevalence of helminthic infections was not different between boys $(27.1 \%)$ and girls (22.8\%) with $P>0.05$. The average intensity of infection was relatively light; 218.0 EPG for hookworms (122.3-261.9 EPG by school), 102.0 EPG for Ascaris (24.0-172.8 EPG), and 36.0 EPG for Trichuris (24.0-48.0 EPG) (Table 2).

\section{Morphological characteristics of hookworm larvae}

Of 100 stool samples, which were cultured by the HaradaMori method, at 10 days after culture, a total of 1,250 filariform hookworm larvae were collected. All (100\%) of the larvae were identified as those of $N$. americanus (Fig. 1). Their
Table 3. Intensity of helminth infections in schoolchildren of 4 primary schools

\begin{tabular}{|c|c|c|c|c|c|c|}
\hline \multirow{2}{*}{$\begin{array}{l}\text { School } \\
\text { code }\end{array}$} & \multicolumn{2}{|c|}{ Ascaris } & \multicolumn{2}{|c|}{ Trichuris } & \multicolumn{2}{|c|}{ Hookworms } \\
\hline & No. $(+)$ & EPG & No. $(+)$ & EPG & No. (+) & EPG \\
\hline 1 & 10 & 117.6 & 1 & 48.0 & 103 & 261.9 \\
\hline 2 & 7 & 51.4 & 1 & 24.0 & 89 & 251.9 \\
\hline 3 & 2 & 24.0 & 1 & 24.0 & 52 & 130.2 \\
\hline 4 & 5 & 172.8 & 1 & 48.0 & 31 & 122.3 \\
\hline Total & 24 & 102.0 & 4 & 36.0 & 275 & 218.0 \\
\hline
\end{tabular}

morphological characters included their body length of 520$580 \mu \mathrm{m}(\mathrm{n}=10)$ not including the sheath, the presence of a dark exclamation mark around the mouth part, short and bent tail $(50-70 \mu \mathrm{m})$, and conspicuous transverse striations on the sheath of the tail region.

\section{Molecular identification of hookworm larvae}

A portion of ITS1 of Vietnamese Necator was sequenced and compared with different N. americanus species from GenBank (Table 3). The results showed a comparison of 185 nucleotides of ITS1 genome between Vietnamese Necator (Veca-VN) and other N. americanus species, including Lao N. americanus (Neca1), Japanese N. americanus (Neca2), unknown N. ameri- 
Table 4. Sequencing of the portion of internal transcribed spacer 1 (ITS1) of different Necator americanus isolates from GenBank compared with $N$. americanus from Vietnam ${ }^{2}$

\begin{tabular}{lllllll}
\hline Notation & Origin & Host & Length & Species & GeneBank & Author \\
\hline Neca-VN & VietNam & Human & $185 \mathrm{bp}$ & Necator americanus ${ }^{*}$ & - & This study \\
Neca 1 & Lao & Human & $185 \mathrm{bp}$ & Necator americanus & LC036565.1 & Hasegawa et al,. (2015) \\
Neca 2 & Japan & Human & $185 \mathrm{bp}$ & Necator americanus & LC036563.1 & Hasegawa et al,. (2015) \\
Neca 3 & - & Gorilla & $185 \mathrm{bp}$ & Necator americanus & JX159772.1 & Hamad et al,. (2014) \\
Neca 4 & Malaysia & Human & $185 \mathrm{bp}$ & Necator americanus & JF960373.1 & Ngui et al,. (2012) \\
Neca 5 & Malaysia & Human & $185 \mathrm{bp}$ & Necator americanus & JF960401.1 & Ngui et al,. (2012) \\
Neca 6 & Malaysia & Human & $185 \mathrm{bp}$ & Necator americanus & JF960388.1 & Ngui et al,. (2012) \\
Neca 7 & Malaysia & Human & $185 \mathrm{bp}$ & Necator americanus & JF960397.1 & Ngui et al,. (2012) \\
\hline
\end{tabular}

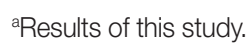

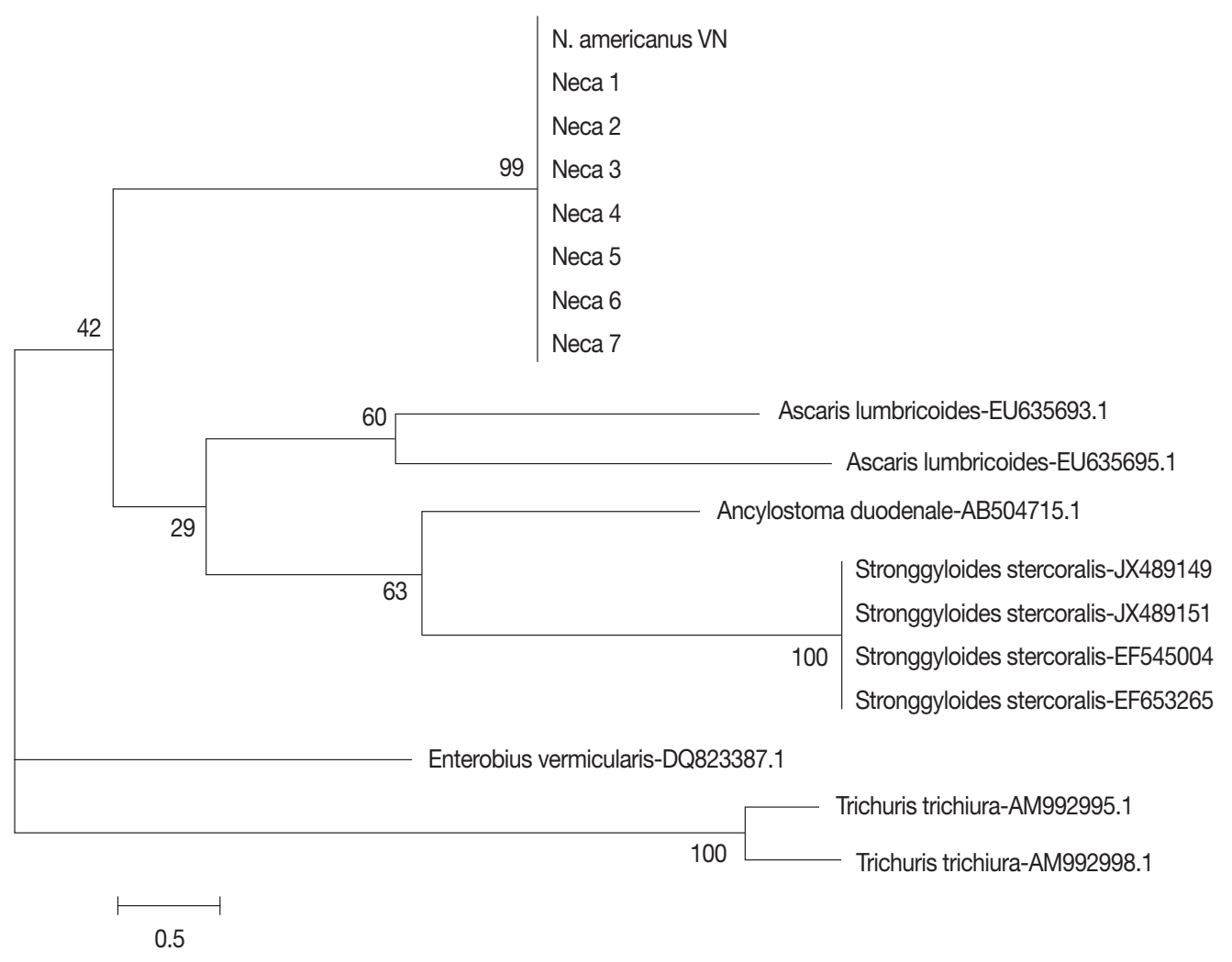

Fig. 2. Phylogenetic tree of Necator americanus Vietnam and other strains from part of ITS1 nucleotide sequence estimated by Neighbor-Joining (NJ) using MEGA 6.06 [12]. Note: N. americanus-VN=Vietnamese Necator; other N. americanus was from GenBank (no. LC036565.1, LC036563.1, JF960397.1, JF960401.1, JF960373.1, JF960388.1, and JX159772.1, respectively); Ascaris lumbricoides (EU635693.1 and EU635695.1); Ancylostoma duodenale (AB504715.1); Strongyloides stercoralis (JX489149, JX489151, EF545004, and EF653265); Enterobius vermicularis (DQ823387.1); Trichuris trichiura (AM992995.1 and AM992998.1).

canus (Neca3), and Malaysian N. americanus (Neca4, Neca5, Neca6, and Neca7) (Table 4). There were no differences between Vietnamese Necator (Veca-VN) and all other species; the homology (similarity) in nucleotides was 100\% (Table 5).

The phylogenetic tree of $N$. americanus Vietnam and other geographical strains derived from partial ITS1 nucleotide sequences estimated by NJ method using MEGA 6.06 [12] showed that the Vietnamese strain of $N$. americanus, together with other geographical strains compared, is one group (Fig. 2).

\section{DISCUSSION}

In the present study, the overall prevalence of helminths was 25.0\%, including 2.0\% Ascaris, $0.33 \%$ Trichuris, and 22.8\% 
Table 5. Percent identity of ITS1 nucleotide sequences of Vietnamese N. americanus and other N. americanus isolates in GenBank

\begin{tabular}{lcccccccc}
\hline & Neca-VN & Neca1 & Neca2 & Neca3 & Neca4 & Neca5 & Neca6 & Neca7 \\
\hline Neca-VN & & 100 & 100 & 100 & 100 & 100 & 100 & 100 \\
Neca 1 & 100 & & 100 & 100 & 100 & 100 & 100 & 100 \\
Neca 2 & 100 & 100 & & 100 & 100 & 100 & 100 & 100 \\
Neca 3 & 100 & 100 & 100 & & 100 & 100 & 100 & 100 \\
Neca 4 & 100 & 100 & 100 & 100 & & 100 & 100 & 100 \\
Neca 5 & 100 & 100 & 100 & 100 & 100 & & 100 & 100 \\
Neca 6 & 100 & 100 & 100 & 100 & 100 & 100 & 100 \\
Neca 7 & 100 & 100 & 100 & 100 & 100 & 100 & 100 \\
\hline
\end{tabular}

Veca-VN is Vietnamese Necator; Neca1 is Lao Necator americanus (GenBank no. LC036565.1), Neca2 is Japanese N. americanus (LC036563.1), Neca3 is unknown origin Necator (JX159772.1), and Neca4, Neca5, Neca6, and Neca7 are Malaysian N. americanus (JF960373.1, JF960401.1, JF960388.1, and JF960397.1, respectively).

hookworms. In comparison with our study, the results of other authors in north Vietnam showed that the prevalence was higher than in our study. For example, in Thai Binh Province (in the north), a study in 1996 targeting a primary schoolchildren revealed that the overall helminthic infection was $74.3 \%$, including 61.0\% Ascaris infection, 45.9\% Trichuris infection, and $1.6 \%$ hookworm infection [10]. Another study in Nghe An Province (in the north) in 2005 showed that the prevalence of overall helminths was $98.0 \%$, including $83.6 \%$ Ascaris, $85.1 \%$ Trichuris, and $30.3 \%$ hookworms [6]. It is difficult to explain the reason why the prevalence of Ascaris and Trichuris was lower in this study; a speculation may include that the soil nature in the subjected area (i.e., sandy soil) may be different from that in previous studies (i.e., clay soil).

In Vietnam, hookworm infections in humans included $A$. duodenale and $N$. americanus, but changed according to the lapse of time. For example, in 1960, hookworm infections included $36.1 \%$ A. duodenale and 54.9\% N. americanus, and 9.0\% mixed-infection with both species [14]. Similarly, in 19681970, hookworm infections included 24.3-34.4\% A. duodenale and $65.6-75.7 \%$ N. americanus [15]. However, in 1981, hookworm infections contained only $3 \% \mathrm{~A}$. duodenale and the majority (97\%) was N. americanus [16]. Similarly, in 1995, hookworm infections included 3.1\% A. duodenale and $96.9 \% \mathrm{~N}$. americanus [6]. Since that time up to now, no reports have shown such information in Vietnam. This present study showed that all 1,250 cultured hookworm larvae obtained from 100 infected schoolchildren were identified as $100 \%$ N. americanus. Comparatively, in Bangladesh, Shahid et al. [9] reported that $A$. duodenale was $11.5 \%$ and N. americanus was $88.5 \%$ in 2010 . In the USA, 95\% of human hookworm cases in 2005 were N. americanus infection [3]. No much information is available from other countries.
The results of the present study may indicate possible disappearance of $A$. duodenale from north Vietnam. The reason is difficult to explain; however, a suggestion could be given as follows: $N$. americanus may be more resistant than A. duodenale to changing environment, including temperature and rainfall, as well as in their susceptibility to anthelmintics such as albendazole, mebendazole, and pyrantel pamoate.

In conclusion, the surveyed area in Krong Pac district, Dak Lak Province, Vietnam was confirmed to be an endemic area of soil-transmitted helminths, particularly, hookworms. The hookworm species determined morphologically after culture of eggs to larvae and molecular analysis of ITS1 sequence was confirmed to be $100 \%$ N. americanus. This may mean disappearance of A. duodenale from north Vietnam. Studies on chronological changes in hookworm species in endemic areas will be helpful for understanding the epidemiological transition of hookworm infections in each area.

\section{ACKNOWLEDGMENT}

This research was funded by Vietnam National Foundation for Science and Technology Development (NAFOSTED) under grant no. 106-YS.05-2014.08.

\section{CONFLICT OF INTEREST}

We have no conflict of interest related to this work.

\section{REFERENCES}

1. Bethony J, Brooker S, Albonico M, Geiger SM, Loukas A, Diemert D, Hotez P. Soil-transmitted helminth infections: ascariasis, trichuriasis, and hookworm. Lancet 2006; 367: 1521-1532.

2. Gasser RB, Cantacessi C, Campbell BE. Improved molecular di- 
agnostic tools for human hookworms. Expert Rev Mol Diagn 2009; 9: 17-21.

3. Hotez PJ, Bethony J, Bottazzi ME, Brooker S, Buss P. Hookworm: "the great infection of mankind". PLoS Med 2005; 2: e67

4. Miyazaki I. Helminthic Zoonoses. Tokyo, Japan. International Medical Foundation of Japan. 1991.

5. John DT, Petri WA Jr. Markell and Voge's Medical Parasitology. 9th ed. St. Louis, Missouri, USA. Saunders Elsevier. 2006.

6. De NV, Than PV, Lien PTH, Minh PN, Phuong TK. Medical Parasitology. Textbook for Training Medical Doctor. Hanoi, Vietnam. 2012, pp 144-185.

7. De NV. Study on current status of hookworm infections and efficacy of anthelmintic drugs in 3 plain regions in the North of Vietnam. Ph.D. thesis in Hanoi Medical University, Hanoi, Vietnam. 1995 (in Vietnamese).

8. Thuan L, Manh T, Uoc PB. Assessment of efficacy for deworming of mebendazole in primary school in Nghe An province. J Malaria Parasit Dis Contr 2005; No. 6: 79-88 (in Vietnamese).

9. Shahid SB, Wazib A, Chowdhury A, Shamsuzzaman SM, Mamun KZ. Identification of hookworm species in stool by Harada-Mori culture. Bangladesh J Med Microbiol 2010; 4: 3-4.

10. Phuong VTB. Assessment of current status of intestinal nematodes in primary schools in Dong Hung district, Thai Binh Province. Master Thesis in Hanoi Medical University, Hanoi, Viet- nam. 2001.

11. Sasa M, Hayashi S, Tanaka H, Shirasaka R. Application of testtube cultivation method on the survey of hookworm and related human nematodes infection. Jpn J Exp Med 1958; 28: 129.

12. Romstad A, Gasser RB, Nansen P, Polderman AM, Monti JR, Chilton NB. Characterization of Oesophagostomum bifurcum and Necator americanus by PCR-RFLP of rDNA. J Parasitol 1997; 83: 963-966.

13. Tamura K, Nei M, Kumar S. Prospects for inferring very large phylogenies by using the Neighbor-Joining method. Proc Nat Acad Sci USA 2004; 101: 11030-11035.

14. Tuy DD. Ancylostoma duodenale and Necator americanus infection in Vietnam. Bulletin of Scientific Workings, Vol. 4. National Institute of Malariology, Parasitology, and Entomology. Hanoi, Vietnam (1957-1974). Medical Publication House. 1975, pp 7476 (in Vietnamese).

15. Thai DD, Thinh TV. Hookworm (Ancylostomatidae). Bulletin of Scientific Workings in Vietnam, Vol. 1. Hanoi, Vietnam. ScienTechnological House. 1976, pp 141-161 (in Vietnamese).

16. Long NP, Lam KT, Dang DV, Luan PC. Ancylostoma duodenale and Necator americanus infection in some areas in Vietnam, 1981. Bulletin of Scientific Workings. National Institute of Malariology, Parasitology, and Entomology. Hanoi, Vietnam (19801985). Medical Publication House. 1985 (in Vietnamese). 